\title{
Shadow Removal Algorithm Based on RGB Color Space
}

\author{
Bailin Lin and Zheng' an Xiao
}

\begin{abstract}
This paper aims to analyze and discuss shadow removal algorithm based on HSV and RGB color spaces. By subtracting the current image with the use of background image we detect the removal targets in the video. This technique becomes an essential component for the background extraction; in this paper the approach we adopt consist to use the traditional Gaussian background modeling method to extract background. However, due to the impact of light and other effects, all obtained foreground image containing shaded regions, and all moving targets detected automatically will be impacted. To perform the effect related to this issue, we introduce RGB Color data instruction to detect foreground shaded region, and then detecting also the real method of removal targets; this is an effective method.
\end{abstract}

Index Terms-Gaussian background modeling, RGB color space shadow detection.

\section{Shadow Removal Algorithm}

According to the level of research in shadow detection-suppression field, a variety of Shadow Algorithm has been developed by researchers during last decade. In this paper we introduce two shadow removal algorithms: the HSV color space shadow removal algorithm and RGB color spaces shadow removal algorithm. We adopt the RGB color space model to create hybrid Gaussian and avoid the region containing shadow effects. And firstly, according to the Pixel attribute, the algorithm can proceeding by determining pattern matching judgment, and use the Gaussian Mixture model to determine shadow pixel. The experiments show that the shadow removal algorithm can be out performed [1].

\section{A. Shadow Detection Algorithm Research}

Conventionally, shadow removal technique can be divided into two categories which are attribute model and pattern model. Shadow algorithm based attribute model nevertheless is seen as simple operation; however the technique requires robustness and inaccuracy characteristics. This section introduces two models based on shadow removal algorithms: HSV and RGB color space shadow removal algorithm.

\section{B. Shadow Detection Algorithm}

According to different classifications, the main requirements in detection can be divided into a variety of shadow detection algorithms. The difference between shadow detection algorithms can be illustrated by using Prati which has high capacity to organize a variety of algorithms categories as shown in Fig. 1.

Manuscript received May 12, 2016; revised August 8, 2016.

The authors are with School of Physics and Mechanical and Electrical Engineering, Hubei University of Education, China (e-mail: home12345@163.com).

\section{Shadow Detection Algorithm}

Shadows is the result of ray's light (such as sun's rays) in accordance to transmission line to generate straight line; and when shadow is kept off, comparing with the non- kept off state, the Pixel brightness dimmed out automatically, the rest of color tolerance still unmodified and take advantage with the use of HSV color model to detect and remove foreground shaded region [2].

HSV color space is a color based on visual characteristics created in 1978s by Smith A.R. According to HSV color model, the following decision can be used to determine whether the pixel is a foreground shaded region:

$$
S P(x, y)=\left\{\begin{array}{l}
1, \alpha \leq \frac{I_{v}(x, y)}{B_{v}(x, y)} \leq \beta \wedge\left(I_{s}(x, y)-B_{s}(x, y)\right) \leq \tau_{s} \wedge \mid I_{H}(x, y)-B_{H}(x, y) \leq \tau_{H} \\
0, \text { else. }
\end{array}\right.
$$

where $I H(x, y), I S(x, y)$ and $I V(x, y)$ represent a new one image pixel value; $B H(x, y), B S(x, y)$ and $B V(x, y)$ the current value of the background pixel point; $\alpha$ and $\beta$ values the part of $(0,1)$ range which vary with the intensity of the current related shadow; $\tau_{\mathrm{S}}$ and $\tau_{\mathrm{H}}$ the experience values, the ret of values for debugging the current environment. From (1-1) obtained, when pixel $S P(x, y)$ is a shadow point by taking the value 1 , the current pixel $S P(x, y)$ with value equal to 0 can be considered as a non-shadow point.

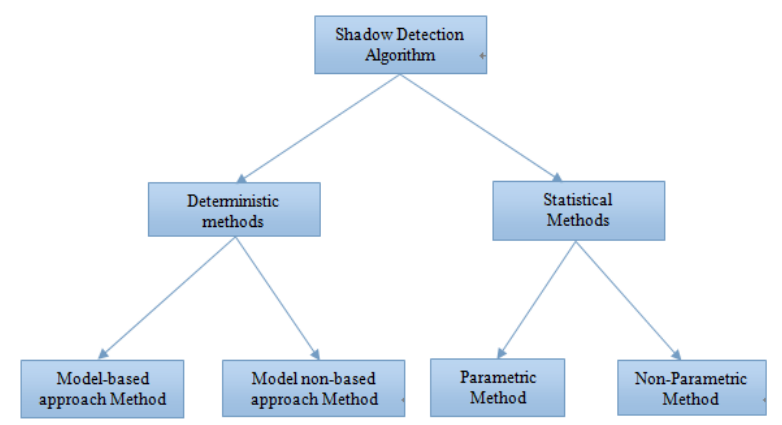

Fig. 1. Shadow detection algorithm classification.

The HSV algorithm above can perform and provide a satisfactory result; but at a higher real-time requirements, the image resulting is detected based on the general RGB color space model; during the shaded based on HSV color space model extraction, it can progressively bring RGB color model to be transformed to HSV color model, and after completing shadow elimination process it is automatically reconverted to RGB color model, which will increase the complexity of operations, and that is not conducive to real-time video image processing sequence.

In summary, this paper propose a quickly shadow removal method, which is a Gaussian mixture RGB color space-based model method. 


\section{A Based RGB Color Space Shadow Removal Algorithm}

\section{1) The RGB shadow model}

It is observed that, the shadow effect changes only the brightness of the current pixel, and consequently the two main aspects of shadow Effect of pixels in the RGB color space can be described as follows: 1) when the current pixel is shaded, the rest of RGB pixels values is smaller than without shadow; 2) when there is a shadow point in the current pixel, the rest of RGB pixels value components contain the same amount of reduction. Therefore, a shadow model based on RGB color space can be then obtained.

$$
\left\{\begin{array}{l}
S_{\mathrm{r}}<\mathrm{B}_{\mathrm{r}}, \mathrm{S}_{\mathrm{g}}<\mathrm{B}_{\mathrm{g}}, \mathrm{S}_{\mathrm{b}}<\mathrm{B}_{\mathrm{b}} ; \\
\mathrm{S}_{\mathrm{r}}: \mathrm{S}_{\mathrm{g}}: \mathrm{S}_{\mathrm{b}}=\mathrm{B}_{\mathrm{r}}: \mathrm{B}_{\mathrm{g}}: \mathrm{B}_{\mathrm{b}}
\end{array}\right.
$$

where $B_{r}, B_{g}$ and $B_{b}$ represent the 3 component values of the current RGB Background pixels; $S_{r}, S_{g}$ and $S_{b}$ represent the current values of detected image sequence RGB pixels.

\section{E. RGB Shadow Detection Algorithm Design}

This algorithm introduces an ideology based on Seed filling, which capable to cope with shadow region and having connectivity issues. Due to the high fluctuation of the B coloration component, therefore, there is no shadow process used to the detection; the following describes the steps of the algorithm implementation (the case study is based on the use of Differential Operators):

1) Entering the original color image, giving an shaded and non-shaded threshold according to the determination values:

$$
f(R)<70 \| f(G)<70 \& \& T>25
$$

2) Use Gaussian - Laplacian operator to carry out R and G color image Algorithm convolution. If the pixel satisfy the step (1) and the threshold determination condition, then from each pixels we got $R=255, G=255$ and $B=0$, the new refilling pixel represent the shaded area; in order to determine all of the pixels and obtain a new image;

3) Obtaining a new repeat image (2) step operation, until the calculation is converged, and obtain the result of shaded region detected.

4) If the result of shaded detected is unsatisfactory the step (1) is modified, and the repeat step (2) and (3) is made until a satisfactory detection result is obtained;

\section{SYSTEM IMPLEMENTATION AND EXPERIMENTAL RESULTS}

In this section, we present the experimental system environment used, including software applications, and introduce systematically the traditional Gaussian background modeling method for background extraction. We use RGB foreground color information detection in shaded region [3].

\section{A. Software}

The software development platform operating system is based in Window7; the VS2010 environment adopts the use of OpenCV2.4.10.

OpenCV include the core library $\mathrm{Cv}$, the auxiliary libraries CvAux, the data structures and linear algebra library CxCore, the library High GUI, the machine learning library ML including the $\mathrm{CV}$ module, which is the basic image processing function and advanced computer vision algorithms. The four OpenCV schematic modules are shown in Fig. 2.

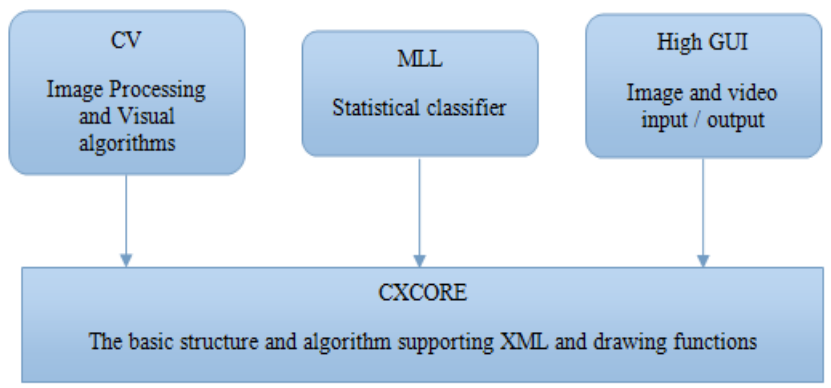

Fig. 2. The basic structure of the open CV.

\section{B. Design Ideas}

\section{1) The Gaussian model}

The removal target detection applications can be divided into two scenarios which are a fixed and non-fixed camera movement; in the context of non-fixed camera movement we observe complex scenes, common optical flow detection, and the complexity of the optical flow computation, restricted scenarios, including the non- extend stat of application. The algorithm detection used in this scenario is a fixed camera which can achieve the scene due to the slowly modifications observed in the background, finally we adopt the use of Gaussian mixture background for the algorithm modeling.

\section{a) Single Gaussian model (SGM)}

We let $\mathrm{X}$ be a Gaussian distribution, the rest of probability density functions (3) are shown in following formula:

$$
N(x, u, \Sigma)=\frac{1}{\sqrt{2 \pi|\Sigma|}} \exp \left[-\frac{1}{2}(x-u)^{T} \Sigma^{-1}(x-u)\right]
$$

where $\mathrm{u}$ represents the expected model, $\Sigma$ is the variation model; we adopt the use of sampling to determine the sample mean value of $\mathrm{u}$ and to determine the value of variance $\Sigma$. In the geometry context, we have adopted respectively Single two-dimensional based on Gaussian distribution and the three-dimensional to perform the ellipse and ellipsoid. However, in some cases, the sample points are not sampled on the ellipse or ellipsoid, and this lack can be performed by the use of Gaussian mixture model.

\section{b) Gaussian mixture model (GMM)}

Gaussian mixture model is based on a single Gaussian model, and the formula (4) shows the relationship between Gaussian mixture model and a single Gaussian model:

$$
\operatorname{Pr}(x)=\sum_{K=1}^{K} \pi N\left(x, u_{k}, \Sigma_{k}\right)
$$

where $\mathrm{K}$ is a preset value. From the formula, Gaussian mixture model is a set of weights and a single Gaussian distribution model, wherein the single Gaussian distribution called Gaussian mixture elements. It should be noted that the reason for using a single Gaussian distribution model as an element mixed Gaussian distribution is due to is better 
computing performance single Gaussian function; it is a good application of Gaussian mixture distribution model.

In the practical application, establishing Gaussian mixture model require division into two cases: the first is the clear sampled sample classification, the second one is the classification of unknown samples. And as it is known for the sample classification, the value of the formula (5) in $\pi_{\mathrm{k}}$ can easily make the determination by using the following formula:

$$
\begin{gathered}
\pi_{k}=\frac{N_{k}}{N} \\
u_{k}=\frac{1}{N_{k}} \sum_{X \in L(k)} X \\
\sum_{k}=\frac{1}{N_{k}} \sum_{x \in L(k)}\left(x-u_{k}\right)\left(x-u_{k}\right)^{T}
\end{gathered}
$$

For the classification of unknown samples, the $N_{k}$ value is unknown, and it can be determined by the following formula:

$$
\begin{gathered}
N_{k}=\sum_{i=1}^{N} \gamma(i, k) \\
u_{k}=\frac{1}{N_{k}} \sum_{i=1}^{N} \gamma(i, k) x_{i} \\
\sum_{k}=\frac{1}{N_{k}} \sum_{i=1}^{N} \gamma(i, k)\left(x_{i}-u_{k}\right)\left(x_{i}-u_{k}\right)^{T} \\
\pi_{k}=\frac{N_{k}}{N}
\end{gathered}
$$

In the purpose to adapt a better performance in light slowly case, Gaussian can mix the model to the center position and the value of the current pixel Gaussian distribution comparison and update the current position by selecting the center of a certain weighting factor. in the case of implication, the problem of light are unsolved in the cases of Gaussian mutation, flowing clouds and shadows. We should believe that a shadow or cloud float does not belong to the content of the background modeling.

Gaussian mixture background is a Gaussian distribution model, and if the prospect of data aggregation can satisfy a certain level, people can still be seen as a new Gaussian distribution; that is means stopping to consider the item (the person) as a new Gaussian distribution item, which even is once in slow motion, that motion is difficult to form a Gaussian distribution. Then, illustrations above indicate that Gaussian mixture model is an adapted technique to detect slow moving items.

Open CV's cvaux library provides an implementation of Gaussian mixture model function. Gaussian mixture background modeling used in this paper is based on the OpenCV library to achieve the mainly following functions:

1) Cv Gauss BG Model:
It is a class that contains a variety of parameters of the model;

2) cv Create Gaussian BG Model:

It set up the property to establish the parameters of the Gaussian mixture distribution model;

3) cv Release Gaussian BG Model and cv Update Gaussian BG Model:

For setting the background model initialization parameters and updates.

Theoretically, Gaussian mixture background modeling is a reliable and perfect backdrop for segmentation techniques. However, in consideration of interference light in the pixel values during short periods at a central location, this lack does not make the data fluctuate around this position normalized by Gaussian distribution center. And for this reason, it is impossible to get accurate center value and variance of data, which will result in an non-perfect segmentation.

\section{The Based RGB Shadow Detection}

We adopt the use of a video recorder outdoors detection system, where the light source position is very far from Earth and the sun, and the light emitting source is seen as parallel light, which can ignore the half shadow penumbra umbra effect [4]. The brightness and color information of the shadow areas are quietly different from the background region. Kai-Tai Song had proposed a hypothesis and makes a proof that there are three components of the RGB ratio and light regions corresponding to the shaded area within a certain period of time, and that can be regarded as constant time. And the above theory can be represented by the following formula:

$$
R_{\text {shadow }}=\mathrm{aR}_{\text {light }}, \mathrm{G}_{\text {shadow }}=\mathrm{bG}_{\text {light }}, \mathrm{B}_{\text {shadow }}=\mathrm{gB}_{\text {light }}
$$

where $R_{\text {shadow }}, G_{\text {shadow }}, B_{\text {shadow }}, R_{\text {light }}, G_{\text {light }}$ and $B_{\text {light }}$ values represent the same region of shadows and light conditions of RGB values, and $\mathrm{a}, \mathrm{b}$, and $\mathrm{g}$ is a constant less than 1 , in general but not equivalency.

In general case, shadow detection presents errors, therefore the evaluation of environment detection method should be done, and two detection error scenarios have to been considered. A Prati first proposed the use of two variables $\mathrm{h}$ and $\mathrm{x}$ to measure Shadow Detection Method based on pros and cons, the specific definitions can be presented as follow:

$$
\begin{aligned}
& h=\frac{T P_{s}}{T P_{s}+F N_{s}} \\
& X=\frac{T P_{F}}{T P_{F}+F N_{F}}
\end{aligned}
$$

where $T P_{S}$ and $T P_{F}$ represent the number of shaded areas and prospects of regional correctly detected pixels, $\mathrm{FN}_{\mathrm{S}}$ and $\mathrm{FN}_{\mathrm{F}}$ represent the number of shaded area and the foreground area error detection pixels.

\section{The Based RGB Shadow Detection}

According to the analyze above, the use of Gaussian mixture technique based in RGB shading algorithm model 
can remove the shadow, which processes is shown in Fig. 3:

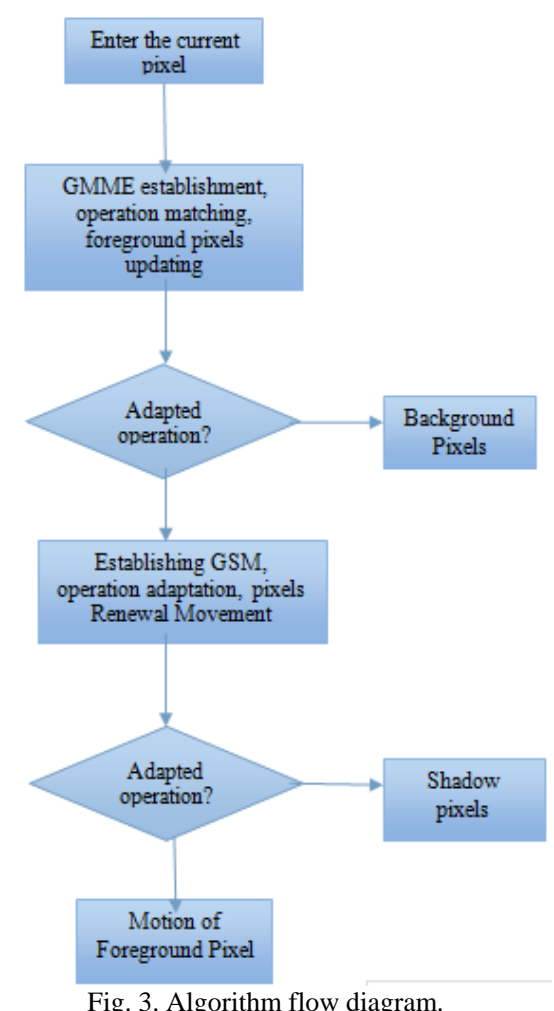

The experimental results obtained concern a video image of the original picture, with difference background foreground images, and the Gaussian mixture model background updated including removal foreground image based on the RGB color space shaded renderings. In order to achieve satisfy the background modeling and shadow modeling time results in this study; we set up 3 Gaussian distribution numbers. The experiments conducted are based on prospects Fig binary processing, and the threshold set up is equal to 30 .



Fig. 4. Fig foreground picture and background subtraction.

\section{1) Contour Tracking}

Contour tracking information pass through the border point of the image, OpenCV contour tracking function is implemented mainly with cvFindContours, cvDrawContours, and the main image contours detected by the contour information stored. The contours stored information transmitted in the form of a pointer is stored to storage location. And that function also includes contour detection mode, a saving method contour, including Offset and other information. And the former is mainly used to describe the internal and external contour outline. The foreground and background subtraction obtained in Fig original contrast is shown in Fig. 4.

2) Using background subtraction target technique for detecting the framework, and use the background parameter model to approximate the background image pixel value, use the differential current frame and the background image in order to achieve the detection process, the background modeling and background update are critical difference.

The different background frame resulting is shown in Fig. 5.

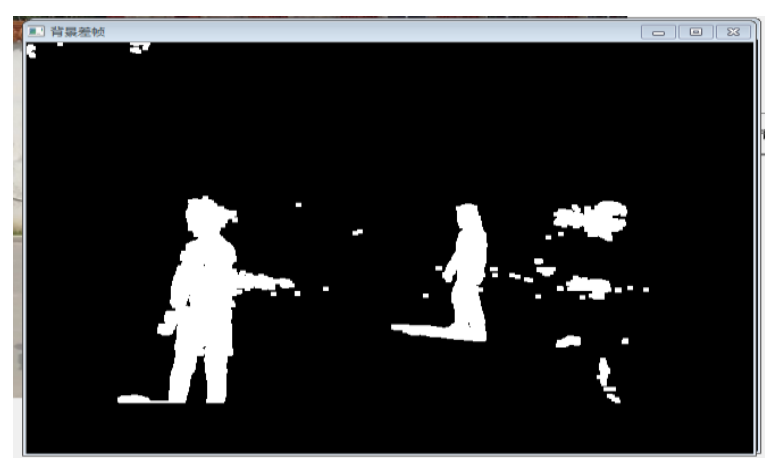

Fig. 5. The different fig background frame.

The based RGB shadows removal algorithm, in shadow removing process while ensuring contour of moving objects without distortion, with a significant inhibited noise scenario, and a real-time detection process to achieve the required in term of speed.

Removing shadow effect diagram, as we can see in Fig. 6.

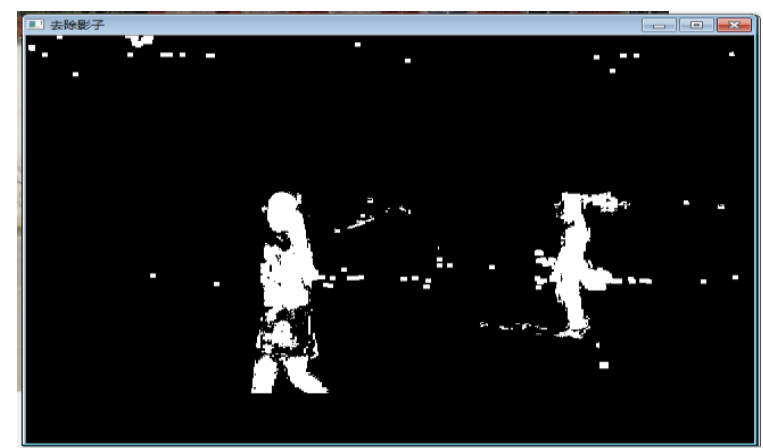

Fig. 6. Shadow removing effect contour.

We use of shadow pixels in the RGB color space characteristics, The shadow model and the current pixel RGB space under the adaptation operation, and then we introduce The Gaussian mixture model, the establishment of Gaussian mixture shadow model corresponding to the foreground, removing process to meet the current shadow pixel Gaussian mixture shadow model, obtained better target detection, we can observe that this shadow removal method works perfectly, an applicable common shadow scenario, with real-high time performance. This research results can be used in various areas of intelligent traffic monitoring.

\section{SUMMARY AND OUTLOOK}

This section give the overview of the work done on this article, and according to the results obtained, the analysis of the advantages and disadvantages of the used method, and 
then process to the forward outlook and prospects for future research [5].

\section{A. Conclusion}

This paper studies the shadow removal algorithm. In this design, the paper has firstly introduced the conventional detection and tracking algorithm detection technique, the ideas based on principle of algorithm detection methods have been explained in detail to understand the source and origin of the work. By comparing the advantages and disadvantages of various suitable algorithms we defined reliable environment detection to set up algorithm processes, and the experiments have verified the effectiveness of our selected algorithm.

\section{B. Future Prospects}

This paper, although it was been used dynamic and adapted background to change slow light adaptation algorithm, However, due to movement of the moving target when the target is similar to the gray background and occlusion, in the purpose to detect problems occurred during undetected process, that constitute one of the issues for further research.

In this paper, we only use RGB image information to calculate the shadow detection, and the shadow detection algorithm was based on the simple RGB color space in the purpose to evaluate the complexity scenario. to satisfy the requirement, the detection algorithm must firstly follow-up sampling calculation, and how to got an automatic transformed algorithm from the current detection algorithm used, without need a pre-sampling process from the video, this issue will constitute our focus point in the future work.

\section{REFERENCES}

[1] S. Elhabian, K. El-Sayed, and S. Ahmed, "Moving object detection in spatial domain using background removal techniques-state-of-art," Recent Patents on Computer Science, 2008, vol. 1, no, 1, pp. 32-54.

[2] O. Barnich and M. Droogenbroeck, "ViBe: A powerful random technique to estimate the background in video sequences," in Proc. 2009 IEEE International Conference on Acoustics, Speech and Signal Processing, 2009, pp. 945-948.

[3] M. Droogenbroeck and O. Paquot, "Background subtraction: Experiments and improvements for ViBe," in Proc. 2012 IEEE Computer Society Conference on Computer Vision and Pattern Recognition Workshops, 2012, pp. 32-37.

[4] T. Yi, W.-M. Liu, and L. Xiong, "Improving robusstneas and accuracy in moving object detection using section-distribution back-ground model[C]," in Proc the 4th International Conference on Natural Computation, 2010, pp. 167-174.

[5] A. K. Shahade and G. Y. Patil, "Efficient shadow removal technique for tracking human objects," Department of Information Technology Shri Sant Gajanan Maharaj College of Engineering, Shegaon, Maharashtra, India, 2014.



Bailin Lin was born in Wuhan-China in June 1971. He received his BS degree in engineering at Wuhan Institute of Water and Transportation in 1992. And he got an MS degree in information engineering at Wuhan University of Technology, where he is currently professor. His current research area include: information engineering, image processing, Microcontroller Applications and Automatic. He has rich professional career and possess several journal and conference publications articles in area of image processing.

Now he is working in School of Physics and Mechanical and Electrical Engineering, Hubei University of Education as a teacher. 